(c) 2019 Universidad Nacional Autónoma de México, Facultad de Estudios Superiores Zaragoza.

Este es un artículo Open Access bajo la licencia CC BY-NC-ND (http://creativecommons.org/licenses/by-nc-nd/4.0/).

TIP Revista Especializada en Ciencias Químico-Biológicas, 22: 1-12, 2019.

DOI: 10.22201/fesz.23958723e.2019.0.177

\title{
Candidatus Liberibacter solanacearum patógeno vascular de solanáceas: Diagnóstico y control
}

\author{
Juan Carlos Delgado-Ortiz ${ }^{1}$, Mariana Beltrán-Beache ${ }^{2}$, Ernesto Cerna-Chávez ${ }^{3}$, \\ Luis Alberto Aguirre-Uribe ${ }^{3}$, Jerónimo Landero-Flores ${ }^{3}$, \\ Yolanda Rodríguez-Pagaza ${ }^{3}$ y Yisa María Ochoa-Fuentes ${ }^{3 *}$ \\ ${ }^{1}$ Catedrático CONACyT-UAAAN, Departamento de Parasitología, Universidad Autónoma Agraria \\ "Antonio Narro", Buenavista, Saltillo, Coahuila 25315, México, ${ }^{2}$ Investigación y Desarrollo, \\ CULTA, S.A. de C.V., Cd. Mante, Tamaulipas 89880, México, ${ }^{3}$ Departamento de Parasitología, \\ Universidad Autónoma Agraria Antonio Narro. E-mail: *yisa8a@yahoo.com
}

\begin{abstract}
RESUMEN
Candidatus Liberibacter solanacearum (CLso) es una bacteria fitopatógena Gram-negativa, limitada al floema en solanáceas y no cultivable in vitro. Es transmitida de manera vertical y horizontal por el psílido Bactericera cockerelli. En México se asocia como responsable de la enfermedad "permanente del tomate", "punta morada de la papa" (Zebra chip) y "variegado del chile". Los síntomas causados por la bacteria varían según el cultivar y la etapa de crecimiento del hospedante pero consisten principalmente en amarillamientos y deformación de la lámina foliar, debido a la alimentación del vector y la colonización del patógeno. Las infecciones ocasionadas por CLso reducen la calidad del producto y el valor comercial en el mercado. La presencia de esta bacteria ha sido detectada en los estados de Coahuila, Sinaloa y Guanajuato, México a través de técnicas moleculares; mientras que el control de la enfermedad se encuentra enfocado en el vector, mediante prácticas culturales y la aplicación de agentes químicos y biológicos. Por lo anterior el objetivo del trabajo es puntualizar la situación actual de la distribución de CLso en México, los métodos de diagnóstico y las estrategias para el manejo integrado de la enfermedad y el vector.
\end{abstract}

Palabras Clave: CLso, sintomatología, manejo, distribución, Zebra chip.

Candidatus Liberibacter solanacearum vascular pathogen of solanaceae: Diagnosis and control

\begin{abstract}
Candidatus Liberibacter solanacearum is a Gram-negative bacterium, obligate parasite of the phloem in Solanaceus crops, not cultivable in vitro. It is transmitted vertical and horizontally by Bactericera cockerelli. In Mexico, CLso is a pathogen asociated with the diseases "permanente del tomate", "punta morada de la papa" (Zebra chip) and "variegado del chile". The symptoms caused by CLso depend on the farming and the stage of growth of the host but it mainly consists in yellowing and deformation of the leaf blade, due to the feeding of the vector and the colonization of the pathogen. Infections caused by CLso reduce the quality and the commercial value of the product in the market. The presence of this bacterium has been detected in the states of Coahuila, Sinaloa and Guanajuato Mexico through molecular techniques, the control of the diseases is focused on the control of the vector, by means of cultural practices and the application of chemical and biological agents. Therefore, the objective of this work is to stand out the current situation of the distribution of CLso in Mexico, the diagnostic methods and the strategies for the integrated management of the disease and the vector.
\end{abstract}

Key Words: CLso, symptomatology, management, distribution, Zebra chip.

Nota: Artículo recibido el 23 de noviembre del 2018 y aceptado el 10 de junio del 2019. 


\section{INTRODUCCIÓN}

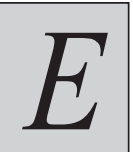

n el estado de Guanajuato se reportó por primera vez en 1984, la existencia de una enfermedad denominada "permanente del tomate" (PT), que causó $60 \%$ de daños en la producción del cultivo (Garzón, 1984); posteriormente, Secor \& Rivera (2004) la relacionaron con una bacteria no cultivable, identificada como Candidatus Liberibacter solanacearum (CLso) en papa (Solanum tuberosum), como el principal agente causal de la enfermedad "zebra chip" (ZC) (en conjunto con algunos fitoplasmas) (Rubio, et al., 2006; Munyaneza, Crosslin \& Upton, 2007a) y del "variegado del chile" (Capsicum annuum) en el centro de México (Munyaneza, Sengoda, Garzón \& Cárdenas, 2009b). Las infecciones ocasionadas por CLso reducen drásticamente la calidad del producto y su valor comercial en el mercado. Los costos por el manejo de la enfermedad y su vector han causado pérdidas millonarias en la compra de ingredientes activos y su aplicación (Gudmestad \& Secor, 2007; Greenway \& Rondon, 2018).

Ravindran, Lévy, Pierson \& Gross, (2015) reportan que la resistencia genética a ZC no había sido identificada en cultivos de papa comerciales, por lo que el manejo de la enfermedad y el desarrollo de materiales resistentes depende de la detección temprana y confiable del agente patógeno, dentro del insecto vector y los tejidos vegetales infectados; sin embargo, la detección de CLso en plantas inmediatamente después de la infección representa un problema debido a los bajos niveles de concentración de este patógeno, lo que dificulta no sólo una eficiente detección por técnicas moleculares, sino que además su aparición se observa varias semanas después (Lévy et al., 2015). CLso se ha reportado en diversas partes del mundo, principalmente en las zonas productoras de papa y en México, los principales cultivos que se han visto afectados son papa, chile y tomate (Solanum lycopersicum); por lo anterior, la presente revisión se centra en la situación actual de su distribución en México, los métodos de diagnóstico de CLso y las estrategias para el manejo integrado de la enfermedad y el vector.

\section{DESCRIPCIÓN DE Candidatus LIBERIBACTER SOLANACEARUM}

CLso es una bacteria Gram-negativa, parásita obligada del floema no cultivable in vitro (Jagoueix, Bové \& Garnier, 1994; Bové, 2006). La morfología del género Candidatus Liberibacter es de un bacilo visto bajo el microscopio electrónico (Tanaka et al., 2007), mide aproximadamente de $2-3 \mu \mathrm{m}$ de largo y $0.2-0.3 \mu \mathrm{m}$ de ancho. Al igual que otras bacterias del género Liberibacter, se puede transmitir por injerto, semilla e insectos vectores (Liefting et al., 2009a; Secor et al., 2009; Crosslin \& Munyaneza, 2009; Camacho-Tapia et al., 2011; Bertolini et al., 2015). Estudios con metagenómica revelaron que el genoma de CLso es un cromosoma circular de 1, 258, $278 \mathrm{pb}$, cuya función de sus genes se estableció por comparación de genes ortólogos. Dentro del genoma se encuentran tres operones completos de rRNA (16s, 23s y 5s), 45 genes que codifican tRNA y alrededor de 35 pseudogenes (Lin et al., 2011).

\section{Distribución DE Candidatus LiberibaCTER SOLANACEARUM}

La distribución geográfica de CLso se basa esencialmente en la presencia de síntomas de ZC, cuyos reportes datan desde 1984 (Secor \& Rivera, 2004), la detección de este patógeno ha sido confirmada en México, EE.UU., Guatemala, Honduras (reporte en Solanum melongena y Nicotiana tabacum), Nicaragua y Nueva Zelanda (Munyaneza, 2012; Munyaneza, Sengoda, Aguilar, Bextine \& McCue, 2013; Aguilar, Sengoda, Bextine, McCue \& Munyaneza, 2013a, 2013b; EPPO, 2013); en México, es considerado como uno de los agentes causales de la enfermedad PT, "punta morada de la papa" (ZC) y daños en chile (Munyaneza, Crosslin \& Upton, 2007a, 2009a, 2009b; Santos-Cervantes, ChávezMedina, Méndez-Lozano \& Leyva-López, 2008; CamachoTapia et al., 2016). Recientemente se reportó la presencia de CLso en muestras de tomate, chile y papa en los estados de Aguascalientes, Baja California, Chihuahua, Coahuila, Edo. de México, Guanajuato, Jalisco, Michoacán, Nayarit, Nuevo León, San Luis Potosí, Sinaloa, Sonora y Zacatecas (Melgoza et al., 2018).

En la actualidad, se han reportado seis haplotipos (Lso) Lso A, Lso B, Lso C, Lso D, Lso E, Lso U, identificados en diferentes cultivos infectados (Munyaneza, Fisher, Sengoda \& Garczynski, 2010; Alfaro-Fernández, Siverio, Cebrián, Villaescusa \& Font, 2012; Lin et al., 2012; Teresani et al., 2014; Haapalainen et al., 2018). Lso A y Lso B se transmiten por el psílido Bactericera cockerelli (Bc), y se asocian con ZC en papa y a otras enfermedades en solanáceas en América, mientras que en Nueva Zelanda sólo se ha registrado la presencia de Lso A. Los haplotipos Lso C y Lso D se transmiten por los psílidos de la zanahoria (Daucus carota) Trioza apicalis y Bactericera trigonica reportadas en Europa, el haplotipo E se identificó recientemente en España afectando apio y zanahorias (Munyaneza, Fisher, Sengoda \& Garczynski, 2010; Liefting, Pérez Clover \& Anderson, 2008, 2009a, 2009b; Camacho-Tapia et al., 2016; AlfaroFernández et al., 2012; Teresani et al., 2014; Rojas-Martínez et al.,2016) e igual sucedió con el haplotipo Lso U, detectado en Trioza urticae y en la planta Urtica dioica, este haplotipo está estrechamente relacionado a los haplotipos Lso A y D (Haapalainen et al., 2018).

En México, se han reportado infecciones con los haplotipos Lso A en plantas de chile y tomate colectadas en el estado de Sinaloa, Lso B en papa en los estados de Chihuahua y Coahuila (Nelson, Tonja, Fisher \& Munyaneza, 2011), Toluca, Edo. de México además de chile e insectos (Rojas- 
Martínez et al.,2016). Ambos haplotipos, se han registrado en la Región Centro del país, en los estados de Michoacán y en el Estado de México en muestras de plantas de chile e insectos de Bc.

\section{VECTOR Bactericera cockerelli}

El psílido de la papa y/o del tomate Bc, también llamada paratrioza o salerillo, es una plaga de importancia económica que daña a las plantas cuando se alimenta, a través del aparato bucal tipo picador-chupador, que está armado con un estilete formado por dos conductos. En la planta, las ninfas y/o adultos introducen el estilete hasta el floema; por uno de los conductos el insecto succiona la savia y por el otro inyecta saliva a la planta. El daño directo por la alimentación de éste, induce el amarillamiento de las hojas, entrenudos cortos y engrosados, retraso del crecimiento de las plantas y reducción en el tamaño del fruto; además de los daños indirectos por la transmisión de los fitopatógenos (Garzón, Velarde, Marín \& Cárdenas, 2005; Nachappa, Lévy \& Tamborindeguy, 2012; Swisher, Munyaneza, Velásquez-Valle \& Mena-Covarrrubias, 2018); uno de los principales patógenos que transmite Bc es CLso (Secor et al.,2009; Liefting, Weir, Pennycook \& Clover, 2009b), lo cual contribuye a incrementar la severidad de los síntomas por la alimentación del insecto debido al consumo de nutrientes de la planta, la multiplicación de la bacteria y la expresión de factores de virulencia del patógeno. Tanto el psílido (Bc) como la bacteria (CLso) tienen el rango de hospederos, siendo los principales las solanáceas (Liefting, Weir, Pennycook \& Clover, 2009b).

La transmisión de CLso por Bc es de forma vertical (Casteel, Hansen, Walling \& Paine, 2012) y horizontal en camote (Ipomoea batatas) y enredadera silvestre (Convolvulus arvensis) (Torres et al., 2015), con período de incubación de hasta $24 \mathrm{~h}$ y tiempo de transmisión promedio de $15 \mathrm{~min}$, no requiere de un hospedante silvestre para multiplicarse, como es el caso de algunos virus fitopatógenos. La edad fisiológica del vector no influye en la capacidad para transmitir la bacteria; sin embargo, con Bc las ninfas requieren menor tiempo (15 min) que los adultos (30 min) para adquirir CLso (Garzón et al., 2009), todos los estadios de Bc transmiten CLso y la transmisión vertical por las hembras adulto puede ser variable de 46.7- 87.5\% (Casteel, Hansen, Walling \& Paine, 2012).

$\mathrm{Bc}$ mantiene una relación simbiótica con la CLso, siendo este endosimbionte el que coloniza el intestino del vector y le ayuda a desdoblar carbohidratos para su fácil asimilación. Además, CLso se involucra en el proceso de desarrollo de los psílidos, como la activación de genes implicados en el metabolismo, reproducción, desarrollo, activación de la respuesta al estrés y la respuesta inmune (Nachappa, Lévy \& Tamborindeguy, 2012). CLso es un simbionte hereditario en Bc (Baumann, 2005).

\section{Proceso de Patogénesis de Candidatus LiBERIBACTER SOLANACEARUM}

El proceso de patogénesis de CLso ha sido establecido de forma hipotética basado en los componentes encontrados en el genoma de la bacteria. Se ha determinado que CLso utiliza fuentes extracelulares de ADP/ATP mediante un transportador tipo NttA (COG3202) (Lin et al., 2011), que le permite utilizar las moléculas de energía del hospedante, ya que carece de un sistema fosfotranferasa para el transporte de azúcares en la membrana interna, pero codifica un transportador de la familia de las permeasas de fucosa (COG0738), que le permite introducir azúcares desde que la bacteria coloniza los tejidos del floema de la planta, donde tiene acceso a sacarosa, fructosa y glucosa (Viola, 2001; Karley, Douglas \& Parker, 2002). El genoma de CLso también codifica un transportador de dicarboxilato (COG1301) de la familia de DetA, los miembros de esta familia transportan una amplia gama de sustratos, incluyendo succinato, oxaloacetato, fumarato y malato (Yurgel \& Kahn, 2004; Groeneveld, Oude, Weme, Duurkens \& Slotboom, 2010). Estos cuatro compuestos, en particular el malato, pueden ser utilizados por la bacteria en la respiración como fuente primaria de carbono, además de la glucosa durante la colonización de la planta (Lin et al., 2011).

El sistema de secreción Tipo I (T1SS) es utilizado por bacterias patógenas para el transporte de toxinas y otras moléculas. T1SS consta de un transportador tripartito que forma un canal contiguo a través de las membranas interna y externa de la bacteria (Linhartová, et al., 2010; Shrivastava \& Miller, 2009). Se han identificado esos tres componentes en el genoma de CLso HlyD (COG0845), PrtD (COG4618) y un pariente lejano de TolC (COG1538). Los genes ortólogos HlyD y PrtD se encuentran juntos dentro del genoma y agrupados con una toxina $r t x$ (COG2931) (Lin et al., 2011); la serralisina junto con algunas proteasas son importantes factores de virulencia, segregadas al medio a través de sistemas de secreción como el Tipo I encontrado en el genoma de CLso (Lin et al., 2011); Ravindran et al. (2017) demostraron que el gen putativo de serrralisina en CLso no expresa su actividad pectolítica en mutantes de Escherichia coli y Serratia liquefaciens, mientras que en la planta de tomate los niveles de serralisina aumentan gradualmente con el avance de la infección, por lo que asociaron este comportamiento a la expresión del gen CKC_02265 que puede estar relacionado a la patogenicidad y a la sobrevivencia de CLso en la planta hospedante. Yao et al. (2016) reportaron la presencia de 16 genes putativos efectores del sistema de secreción tipo IV, cuatro de las cuales codifican para las proteínas de ensamble pilus diferencialmente expresadas por los haplotipos Lso A y Lso B, sistema que se asocia a un factor de virulencia que juega un papel en el reconocimiento; así como su participación en la patogenicidad especifica de los haplotipos. 
Otro grupo de genes que participan en el proceso de patogénesis, son los implicados en la absorción y retención de tiamina y hierro como ftr 1 , asociado con los diversos niveles de virulencia en otros organismos (Nyilasi, 2008). Factores como la $n$ tta y transportadores ftr 1 podrían estar implicados en el desarrollo de la enfermedad, causando el agotamiento de la energía y deficiencia de nutrientes en la planta (Lin et al., 2011).

\section{TÉCNICAS DE DIAGNÓSTICO Síntomas}

Los síntomas causados por CLso en los cultivos que infecta varían según el cultivo y la etapa de crecimiento del hospedante (Khairulmazmi, Kamaruzaman, Habibuddin, Jugah \& Syed, 2008). En papa, las plantas infectadas muestran retraso en el crecimiento y clorosis, sobrecrecimiento de los entrenudos, proliferación de yemas axilares, brote de tubérculos aéreos (Figura 1), oscurecimiento del sistema vascular y muerte temprana de la planta (Munyaneza, Crosslin \& Upton, 2007a). Se ha estimado en un 20-40\% que los tubérculos infectados por ZC generalmente no brotan y si lo hacen producen brotes ahilados y plantas débiles (Henne et al., 2010); además, estos tubérculos muestran lenticelas agrandadas

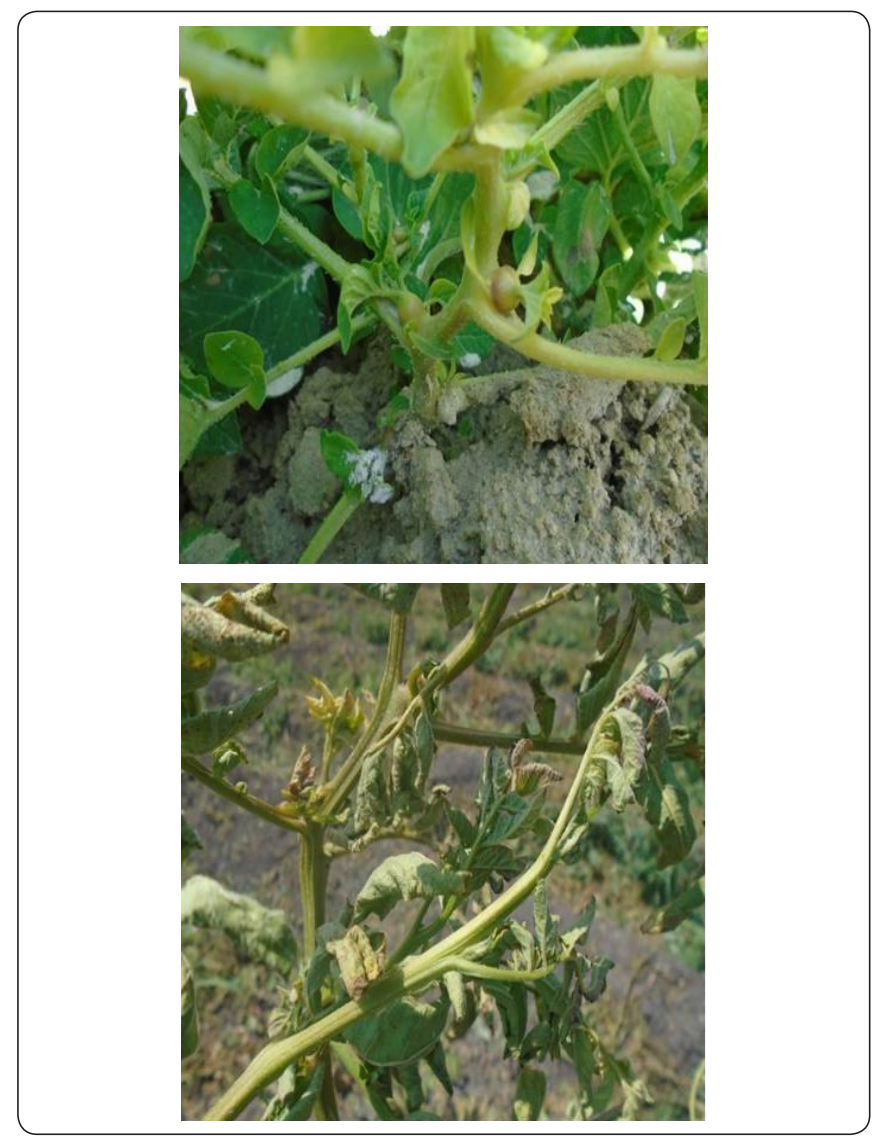

Figura 1. Sintomatología de CLso en plantas de papa (Solanum tuberosum). en el tallo subterráneo, colapso de estolones, lesiones color marrón en el anillo vascular, necrosis en los tejidos internos y ocasionalmente rayado color marrón en el tejido medular (Munyaneza, Goolsby, Crosslin \& Upton, 2007b).

En tomate, dependiendo de la infección, ya sea por los haplotipos Lso A o Lso B, Mendoza-Herrera et al. (2018) reportan la aparición de síntomas como la reducción del crecimiento, reducción del tamaño y decoloración de las hojas apicales, desarrollo de meristemos axilares, enchinamiento, atrofia, enrollamiento, detención del crecimiento y necrosis de los tejidos hasta el colapso de la planta. Las plantas de chile infectadas con CLso muestran crecimiento apical clorótico o color verde pálido, adelgazamiento del ápice y deformación de las hojas, entrenudos cortos, retraso general del crecimiento y el variegado típico (Figura 2) (Munyaneza, Sengoda, Garzón \& Cárdenas, 2009b; Camacho-Tapia et al., 2011).

\section{Diagnóstico Microscópico}

La morfología del género de Candidatus Liberibacter se observa en forma de bacilo bajo el microscopio electrónico, de $2-3 \mu \mathrm{m}$ de largo, $0.2-0.3 \mu \mathrm{m}$ de ancho, con extremos redondeados y con una superficie exterior suave e indica que no poseen una membrana externa, por lo que la pared de los peptidoglicanos es apenas visible (Tanaka et al., 2007), haciéndola negativa a la tinción de Gram. Cicero, Fisher \& Brown (2016), con disecciones y extracción de la bacteria del intestino medio del psílido, determinaron la presencia de pili y flagelos e igual en el genoma de CLso se han observado regiones codificantes para proteínas de ensamble de pili y flagelos (Lin et al., 2011). Su citoplasma es menos denso con respecto a otras bacterias fitopatógenas y con frecuencia muestran plasmólisis (Jagoueix, Bové \& Garnier, 1994).

\section{Diagnóstico biológico}

Se han logrado aislar y cultivar in vitro las tres especies de Candidatus Liberibacter que infectan cítricos, en el medio "Liber A" sobreviviendo un lapso de 10 a 14 días, preparado con extracto de venas de cítricos, potasio monobásico, fosfato dibásico, NADP, cicloexamida y agar (Sechler et al., 2009). En el caso especial de CLso se han realizado pruebas de patogenicidad y búsqueda de variedades resistentes a $\mathrm{ZC}$, mediante transmisión directa de CLso por insectos adultos de Bc (Munyaneza, Buchman, Sengoda, Fisher \& Pearson, 2011; Lévy, Ravindran, Gross, Tambrindeguy \& Pierson, 2011; Camacho- Tapia et al., 2016) e injerto de yemas y brotes de crecimiento de plantas cultivadas en campo con síntomas de CLso y a partir de tubérculos sintomáticos, al no contar con un medio sintético para su aislamiento y poder replicar los síntomas de ZC (Secor et al., 2009).

\section{Diagnóstico serológico}

Se han obtenido varios anticuerpos monoclonales (AcM) específicos para Huanglongbing (HLB) en cítricos, usando 


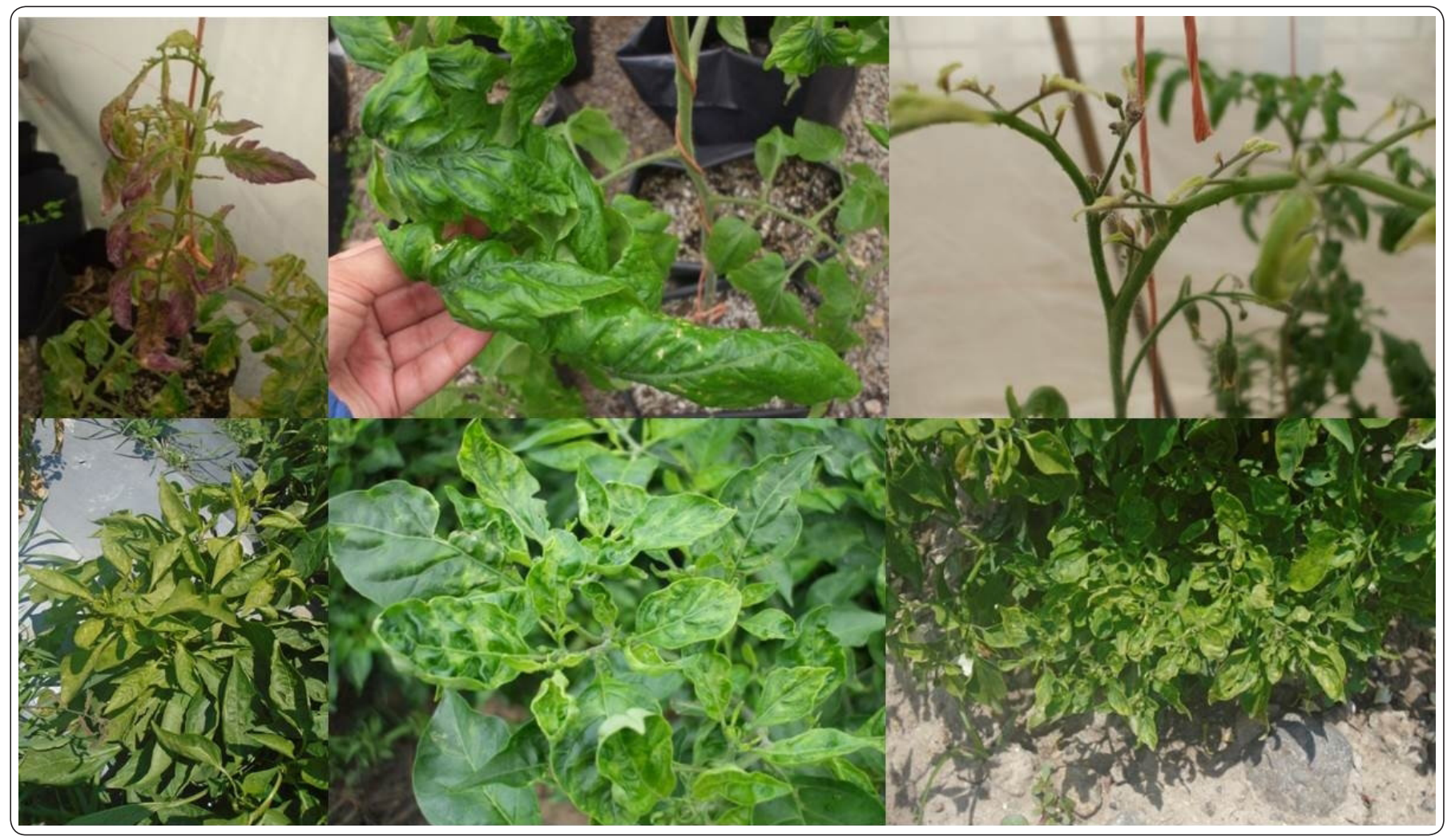

Figura 2. Sintomatología de CLso en plantas de tomate (Solanum lycopersicum) (arriba) y Chile (Capsicum annum) (abajo).

como antígenos cepas asiáticas y africanas (Villechanoux, Garnier \& Bové, 1990; Garnier et al., 1991; Gao, \& Bové, 1991). La producción de AcM no ha sido eficiente para la detección de las diferentes especies del género Liberibacter debido a su alta especificidad respecto a la cepa contra la que son dirigidos. Es por ello que se requiere de un estudio más profundo para la búsqueda de epítopes comunes a todas las formas conocidas de la bacteria, con el fin de generalizar su aplicación en el diagnóstico (Collazo, Pantoja \& Llauger, 2008).

\section{Diagnóstico molecular}

La reacción en cadena de la polimerasa (PCR) es la técnica más utilizada debido a la especificidad y rapidez con que se realiza, la detección mediante PCR de Candidatus Liberibacter está dirigida a la amplificación de la región $16 \mathrm{~S}$ rDNA de su genoma (Jagoueix, Bové \& Garnier, 1994; Texeira et al., 2005). En la Tabla I se muestran los principales iniciadores para el género Candidatus Liberibacter y específicos para la detección de CLso.

\section{LOOP-MEDIATED ISOTHERMAL AMPLIFICATION PROCEDURE (LAMP)}

La técnica LAMP para CLso fue desarrollada por Ravindran, Lévy, Pierson \& Gross (2015), quienes refieren que la técnica muestra la misma sensibilidad que una PCR convencional en pruebas de detección en campo; cabe destacar que es una técnica de rápida detección y con un requerimiento mínimo de equipo; la técnica se basa en la síntesis de DNA de desplazamiento de cadena por autociclado favorecida por la DNA polimerasa de Bacillus stearothermophilus (Bst) y la integración de los iniciadores: LsoTX16SLamp-F3 (CTGCATGGCTGTCGTCA), LsoTX16SLamp-B3c (GTAACCACCATTGTAGC), LsoTX16SLamp-FIP (F1c-F2) (TAGAGGTAGGGGTTGC/ GTGTCGTGAGATGTTGGG), LsoTX16SLamp-BIP (B1B2c) (GGGTACTTTATAGGGA/CCATGAGGACTTGACG), LsoTX16SLamp-LF (GCTCGTTGCGGGACTTA), LsoTX16SLamp-LB (GGAGGAAGGTGGGGATG). Las reacciones con resultados positivos se tornan de color verde fluorescentes bajo iluminación de luz UV, mientras que las muestras negativas mantendrán esa falta de fluorescencias; este efecto se debe a la formación de un pirofosfato de magnesio durante la síntesis de DNA que genera una turbidez fácilmente distinguible en los tubos.

\section{ESTRATEGIAS DE CONTROL}

\section{Control cultural}

Se recomienda eliminar focos de infección como cultivos abandonados; los residuos del cultivo inmediatamente después de la cosecha y la eliminación de plantas hospederas del vector, plantas enfermas en los márgenes del cultivo y lotes adyacentes (Bujanos, Garzón \& Marín, 2005). El uso de 


\begin{tabular}{|c|c|c|c|}
\hline Primer & Secuencia & Organismo & Referencia \\
\hline $\begin{array}{l}\text { *OI2c/ Cli.po.F / } \\
\text { LsoF/ OA2 /ZCf }\end{array}$ & $\begin{array}{l}\text { GCCTCGCGACTTCGCAACCCAT/ } \\
\text { TACGCCCTGAGAAGGGGAAAGATT/ } \\
\text { GTCGAGCGCTTATTTTTAATAGGA/ } \\
\text { GCGCTTATTTTTAATAGGAGCGGCA/ } \\
\text { CGAGCGCTTATTTTTATTAGGAGC }\end{array}$ & $\begin{array}{l}\text { Candidatus } \\
\text { Liberibacter }\end{array}$ & $\begin{array}{l}\text { Jagoueix, Bové \& } \\
\text { Garnier,1994; Secor et } \\
\text { al., 2009; Li et al., 2009; } \\
\text { Liefting et al., 2009; }\end{array}$ \\
\hline $\begin{array}{l}\text { LG774F/ } \\
\text { LG1463R }\end{array}$ & $\begin{array}{l}\text { GTAAACGATGAGTGCTAGCTGTTGGG/ } \\
\text { CTGACCRTACCGTGGCCGG }\end{array}$ & $\begin{array}{l}\text { Candidatus } \\
\text { Liberibacter }\end{array}$ & Morris et al., 2017 \\
\hline Lso adkF/ Lso adk R & $\begin{array}{l}\text { GCGCCACACTAACATCTCCTTCC/ } \\
\text { CGCAGCAGTATGAGGGCC }\end{array}$ & CLso & Ravindran et al., 2011 \\
\hline $\begin{array}{l}\text { Lso TX 16/23 F/Lso } \\
\text { TX 16/23 R }\end{array}$ & $\begin{array}{l}\text { AATTTTAGCAAGTTCTAAGGG/ } \\
\text { GGTACCTCCCATATCGC }\end{array}$ & CLso & Ravindran et al., 2011 \\
\hline $\begin{array}{l}\text { OMB } 1482 \mathrm{~F} \\
\text { /OMB } 2086 \mathrm{R} \\
\text { CLZC-F/ CLZC-R }\end{array}$ & $\begin{array}{l}\text { GGCGTGGTTATAAGCAGAGT/ } \\
\text { ATCTACACGCGGACCTATAC } \\
\text { TCGGATTTAGGAGTGGGTAAGTGG/ } \\
\text { ACCCTGAACCTCAATTTTACTGAC }\end{array}$ & $\begin{array}{l}\text { CLso (Proteína de la } \\
\text { membrana externa de } \\
\text { Liberibacter) }\end{array}$ & $\begin{array}{l}\text { Crosslin, Lin \& } \\
\text { Munyaneza, } 2011\end{array}$ \\
\hline CL514F/ CL514R & $\begin{array}{l}\text { CTCTAAGATTTCGGTTGGTT/ } \\
\text { TATATCTATCGTTGCACCAG }\end{array}$ & $\begin{array}{l}\text { Candidatus } \\
\text { Liberibacter }\end{array}$ & $\begin{array}{l}\text { Munyaneza, Sengoda, } \\
\text { Crosslin, De la Rosa \& } \\
\text { Sánchez, 2009c }\end{array}$ \\
\hline $\begin{array}{l}\text { Lp Frag4-1611F/LP } \\
\text { Frag 4-480R }\end{array}$ & $\begin{array}{l}\text { GGTTGATGGGGTCATTTGAG/ } \\
\text { CACGGTACTGGTTCACTATCGGTC }\end{array}$ & $\begin{array}{l}\text { Candidatus } \\
\text { Liberibacter } \\
\text { psyllaurous }\end{array}$ & $\begin{array}{l}\text { Hansen, Trumble, } \\
\text { Stouthamer \& Paine, } 2008\end{array}$ \\
\hline
\end{tabular}

* Complementarios OI2c/ Cli.po.F; OI2c / LsoF; OI2c /OA2; OI2c /ZCf.

Tabla I. Iniciadores para la detección de Candidatus Liberibacter solanacearum.

acolchados plásticos de colores aluminio y blanco favorecen la disminución de la densidad de poblaciones de $\mathrm{Bc}$ en áreas cultivadas con tomate (Demirel \& Cranshaw, 2006).

\section{Control genético}

El desarrollo de variedades vegetales resistentes o tolerantes, mediante métodos de mejoramiento genético tradicional o ingeniería genética, representan una estrategia de control eficaz y sostenible para el manejo de ZC (Munyaneza, Buchman, Sengoda, Fisher \& Pearson, 2011). Curtis, Tantravahi \& Mirkov, (2010) insertaron un gen anti-insectos y dos genes antimicrobianos en papa, reduciendo el inóculo bacteriano y retardando la aparición de los síntomas por la alimentación del psílido y de CLso sin mostrar niveles de tolerancia con significancia en el control de la enfermedad entre los materiales transgénicos. Recientemente HernándezDeheza et al. (2018) evaluaron dos clones experimentales de papa (T05-20-11 y Bajío143) y un cultivo comercial (Real 14) contra variedades altamente susceptibles (Milagros y Atlantic); dichos cultivos fueron inoculados con el haplotipo Lso B y una combinación entre los haplotipos Lso A y Lso $\mathrm{B}$; los resultados indicaron que el cultivo Real 14 fue el que mostró un menor porcentaje de decoloración del tubérculo que otros cultivos probados después de la inoculación con la mezcla de haplotipos y Lso B. Mientras el clon T05-2011 mostró menor decoloración del tubérculo que el Atlantic, después de la inoculación con los haplotipos; así como menor severidad (síntomas foliares) que los cultivos Atlantic y Bajío 143. Sin embargo, en 2017 Rubio-Covarrubias et al. evaluaron cuatro líneas avanzadas (246, 865, 510 y NAU) que fueron expuestas a la infección de CLso; donde las líneas 246, 865 y 510 mostraron una menor selección por $\mathrm{Bc}$ y una menor oviposición de $\mathrm{Bc}$ en las líneas 510 y 865; así como un menor índice de decoloración en los tubérculos infectados con CLso de los cuatro genotipos experimentales, siendo que el genotipo 510 ha sido plantado a nivel comercial debido a sus buenas características agronómicas y comerciales. Se ha identificado germoplasma de papas silvestres (Solanum tuberosum, $S$. berthaultii, $S$. raphanifolium, $S$. chacoense y $S$. guerreroense con características de antixenosis a Bc (Machida, 2015), de las cuales algunas no muestran infección por CLso en ensayos de no elección, pero que no cumplen con las características agronómicas requeridas para su comercialización; por lo que es necesario el estudio sobre la utilidad de estas variedades en programas de mejoramiento genético, destacando la importancia de que el efecto de antixenosis no garantiza que las variedades tengan la capacidad de no infectarse con CLso (Wang et al., 2017). Lo anterior difiere del trabajo de Lévy \& 
Tamborindeguy, (2014) quienes evaluaron los mecanismos de antibiosis y antixenosis de $S$. habrochaites y concluyeron que la resistencia de la planta no podría ser suficiente para proteger las plantas solanáceas contra la transmisión de CLso, pero podría ayudar a minimizar el riesgo general de transmisión bacteriana al reducir la alimentación o afectar el desarrollo de la población de vectores.

\section{Control biológico}

El uso de hongos entomopatógenos, insectos depredadores y parasitoides, representa una alternativa para el manejo integrado del $\mathrm{Bc}$ en sus diferentes instares. Los hongos Metarhizium anisopliae, Beauveria bassiana e Isaria fumosorosea probados solos o en conjunto con otros insecticidas (Lacey, de la Rosa \& Horton, 2009; 2010) y Paecilomyces fumosoroseus (Bujanos, Garzón \& Marín, 2005) han demostrado ser útiles en el control de Bc causando mortalidad de un 78-99\% en condiciones de laboratorio y campo, siendo eficaces en cualquier fase de desarrollo del insecto (Lacey, de la Rosa \& Horton, 2009; 2010). Entre los enemigos naturales más eficientes se incluyen larvas de crisopa, coccinélidos, geocóridos, anthocóridos, míridos, nábidos y larvas de sírfidos, así como los insectos parasitoides Tamarixia triozae y Metaphycus psyllidis (Compere, 1943; Pletsch, 1947). Algunos productos biorracionales en el manejo de $\mathrm{Bc}$, son kaolín, aceites minerales y de plantas, que han demostrado reducir la alimentación del psílido y la oviposición (Gharalari et al., 2009; Yang et al., 2010; Peng, Trumble, Munyaneza \& Liu, 2011; Butler, Byrne, Keremane, Lee \& Trumble, 2011). Productos comerciales como Sun Spray (aceites esenciales) o Requiem (extracto de Chenopodium ambrosioides) repelen significativamente de (77.2- $95.4 \%$ ) adultos del Bc y disminuye la ovoposición (Yang et al., 2010). A nivel de laboratorio se ha implementado la aplicación del extracto Heliopsis longipes para evaluar su actividad insecticida sobre ninfas de $\mathrm{Bc}$, demostrando eficiencia en la mortalidad de las ninfas que oscilan de un 30$100 \%$ a las $24 \mathrm{~h}$ de haberse realizado la aplicación del extracto etanólico (Beltrán, Cerna, Delgado \& Ochoa, 2015).

\section{Control químico}

El control de CLso se ha enfocado a la reducción de las fuentes de inóculo, como el uso de plantas libres, remoción de plantas sintomáticas y el control químico del insecto para reducir la transmisión de la bacteria (Camacho-Tapia et al., 2016). De acuerdo a estudios realizados por Zhang et al. (2011) la combinación de penicilina G y estreptomicina tiene efecto antimicrobial contra CLso, la penicilina G potásica es asimilada rápidamente por las plantas y posee bajos niveles de fitotoxicidad; cabe destacar que el tratamiento mencionado fue suministrado con intervalos de aplicación cortos y durante periodos de tiempo prolongados, según el autor este mismo tratamiento puede ser viable para el control de CLso en plantas de papa y tomate.
Los insecticidas más comunes para el control de $\mathrm{Bc}$, son thiametoxam, permetrina, esfenvalerato, imidacloprid, cyfluthrin, methamidophos, endosulfan, imidacloprid, disulfoton, phorato, spiromesifen, ciflutrin, omeotato, aldicarb y thiacloprid (Pavlista, 2002). Pyrioproxyfen, pymetrozine y spinosad también son de utilidad en programas de manejo integrado de plagas para Bc (Liu \& Trumble, 2004). La aplicación de azufre en polvo y sales potásicas de ácidos grasos (jabones insecticidas al 2\%) pueden ser útiles contra ninfas, pero el control es poco confiable (Bujanos, Garzón \& Marín, 2005; Cranshaw, 2007). Sin embargo, Cerna, Hernández, Landeros, Aguirre \& Ochoa, (2015), reportaron tasas de resistencia a los insecticidas, endosulfan e imidacloprid en las poblaciones de Bc de las zonas productoras de chile de San Luis Potosí, Aguascalientes y la zona productora de papa en Coahuila y Nuevo León, México. Mientras que, la población de Bc procedente de San Luis Potosí manifestó la mayor tasa de resistencia a la abamectina.

\section{Conclusiones}

Debido a las características biológicas de la bacteria Candidatus Liberibacter solanacearum y su relevancia como plaga de importancia económica, es transcendental implementar estrategias de detección con una alta efectividad y confiabilidad, así como el utilizar todas las herramientas para el desarrollo de programas de manejo de Candidatus Liberibacter solanacearum y su vector. Esta revisión muestra el panorama actual de la distribución de esta bacteria en México, así como una perspectiva de los métodos de diagnóstico y estrategias de manejo disponibles para el control de la enfermedad.

\section{Agradecimientos}

Agradecemos a CONACYT por el apoyo al Proyecto 1048 del programa Cátedras CONACYT.

\section{REFERENCIAS}

Aguilar, E., Sengoda, V.G., Bextine, B., McCue, K.F. \& Munyaneza, J.E. (2013a). First report of "Candidatus Liberibacter solanacearum" on tomato in Honduras. Plant disease, 97(10),1375. DOI: 10.1094/PDIS-04-130354-PDN

Aguilar, E., Sengoda, V.G., Bextine, B., McCue, K.F. \& Munyaneza, J.E. (2013b). First report of "Candidatus Liberibacter solanacearum" on tabacco in Honduras. Plant disease, 97 (10), 1375. DOI: 10.1094/PDIS-04-130453-PDN

Alfaro-Fernández, A., Siverio, F., Cebrián, M.C., Villaescusa, F.J. \& Font, M.I. (2012). 'Candidatus Liberibacter solanacearum' associated with Bactericera trigonica affected carrots in the Canary Islands. Plant Disease, 96, 581-581. DOI:10.1094/pdis-10-11-0878-pdn

Baumann, P. (2005). Biology bacteriocyte-associated endosymbionts of plant sap-sucking insects. Annual 
Review of Microbiology, 59, 155-189. DOI: 10.1146/ annurev.micro.59.030804.121041

Beltrán, B.M., Cerna, C.E., Delgado, O.J.C. \& Ochoa, F.Y.M. (2015). Evaluación de la actividad insecticida de Heliopsis longipes (A. Gray Blake) sobre ninfas de Bactericera cockerelli (Sulc.) (Hemiptera: Triozidae). Investigación y Ciencia de la Universidad Autónoma de Aguascalientes, 66, 12-15. Disponible en linea: https:// www.uaa.mx/investigacion/ revista/archivo/revista66/ Articulo\%202.pdf

Bertolini, E., Teresani, G.R., Loiseau, M., Tanaka, F.A.O., Barb, S., Martínez, C., Gentit, P., López, M.M. \& Cambra, M. (2015). Transmission of 'Candidatus Liberibacter solanacearum' in carrot seeds. Plant Pathology, 64, 276 285. DOI: $10.1111 /$ ppa. 12245

Bové, J.M. (2006). Huanglongbing: a destructive, newlyemerging, century-old disease of citrus. Journal of Plant Pathology, 88, 7-37. DOI: 10.4454/jpp.v88i1.828

Bujanos, M.R., Garzón, J.A. \& Marín, A. (2005). Manejo integrado del pulgón saltador Bactericera (=Paratrioza) cockerelli (Sulc) (Hemiptera:Triozidae) en los cultivos de solanáceas en México. Pp. 93-99, Memorias, Segunda convención mundial del chile. Zacatecas, México.

Butler, C.D., Byrne, F.R, Keremane, M.L., Lee, R.F. \& Trumble, J.T. (2011). Effects of insecticides on behavior of adult Bactericera cockerelli (Hemiptera: Triozidae) and transmission of Candidatus Liberibacter psyllaurous. Journal of Economic Entomology, 104, 586-594. DOI: org $/ 10.1603 / \mathrm{EC} 10285$

Camacho-Tapia, M., Rojas-Martínez, R.I., Zavaleta-Mejía, E., Hernández-Deheza, M.G., Carrillo-Salazar, J.A., Rebollar-Alviter, A. \& Ochoa-Martínez, D.L. (2011). Aetiology of chili pepper variegation from Yurécuaro, México. Journal of Planth Pathology, 93, 331-335. DOI: dx.doi.org/10.4454/jpp.v93i2.1187

Camacho-Tapia, M., Rojas-Martínez, R.I., ZavaletaMejía, E., Rebollar-Alviter, A., Aranda-Ocampo, S. \& Suárez-Espinosa, J. (2016). Biological, ecological, epidemiological and management aspects of Candidatus Liberibacter. Revista Chapingo Serie Horticultura, 22, 5-16. DOI: org/10.5154/r.rchsh.2015.09.021

Casteel, C.L., Hansen, A.K., Walling, L.L. \& Paine, T.D. (2012). Manipulation of plant defense responses by the tomato psyllid (Bactericerca cockerelli) and its associated endosymbiont Candidatus Liberibacter psyllaurous. PLOS ONE, 7, 1-10. DOI: org/10.1371/ annotation/9903158b-c45c-44b9-b152-7ffb5bec0c32

Cerna, C.E., Hernández, B.O., Landeros, F.J., Aguirre, U.L. \& Ochoa, F.Y.M. (2015). Insecticide-resistance ratios of three populations of Bactericera cockerelli (Hemiptera: Psylloidea: Triozidae) in regions of Northern Mexico. Florida Entomologist, 98, 950-953. DOI: org/10.1653/024.098.0322

Cicero, J.M., Fisher, T.W. \& Brown, J.K. (2016). Localization of 'Candidatus Liberibacter solanacearum' and evidence for surface appendages in the potato psyllid vector. Phytopathology, 106, 142-154. DOI: org/10.1094/ PHYTO-04-15-0088-R

Collazo, C.C., Pantoja, M.L. \& Llauger, R.R. (2008). Técnicas empleadas para el diagnóstico del huanglongbing de los cítricos. Revista CitriFrut, 25, 24-32. Disponible en línea: www.actaf.co.cu/revistas/revista_citrifrut/Citrus $\% 20$ 2\%202008/RC_A5_25_2_2008\%20bn.pdf

Compere, H. (1943). A new species of Metaphycus parasite on psyllids. The Pan-PacificEntomologist, 19, 71-73. Diponible en línea: http://biostor.org/reference/224964/ page $/ 1$

Cranshaw, W.S. (2007). Potato or tomato psyllids. Insects Series Home \& Garden. Colorado States University No. 5, 540. Disponible en línea: www.extension.colostate. edu/docs/ pubs/insect/05540.pdf

Crosslin, J.M. \& Munyaneza, J.E. (2009). Evidence that the zebra chip disease and the putative causal agent can be maintained in potatoes by grafting and in vitro. American Journal of Potato Research, 86, 183-187. DOI:org/10.1007/s12230-009-9070-6

Crosslin, J.M., Lin, H. \& Munyaneza, J.E. (2011). Detection of 'Candidatus Liberibacter solanacearum' in the potato psyllid, Bactericera cockerelli (Sulc), by conventional and real-time PCR. Southwestern Entomologist, 36, 125135. DOI: org/10.3958/059.036.0202

Curtis, L.S., Tantravahi, P. \& Mirkov, T.E. (2010). An evaluation of plant-derived antimicrobial and anti-insect genes on reducing zebra chip disease in transgenic potato p. 110-114 In Proceedings of the 10th Annual Zebra Chip Reporting Session, ed. F.Workneh, and C.M. Rush. Dallas, TX (November 7-10, 2010).

Demirel, N. \& Cranshaw, W. (2006). Relative effect of color mulches to patato/tomato psyllid, Paratrioza cockerelli (Sulc) (Homoptera:Psylidae), on garden Tomatoe plants. Journal of entomology, 3(2), 189-193. DOI: 10.3923/ je.2006.189-193.

EPPO (2013). 'Candidatus Liberibacter solanacearum'. EPPO Bulletin, 43(2), 197-201 DOI: 10.1111/epp.12043

Gao, J. \& Bové, J.M. (1991). 16S ribosomal DNA amplification for phylogenetic study. Journal of Bacteriology, 173, 697-703. https://www.ncbi.nlm.nih.gov/pmc/articles/ PMC207061/

Garnier, M., Gao, S.J., He, Y.L., Villechanoux, S., Gander, J. \& Bové, J.M. (1991). Study of the greening organism (GO) with monoclonal antibodies; serological identification, morphology, serotypes and purification of the GO. Pp, 428-435 In: Proceedings of the 11th Conference of the International Organization of Citrus Virologists: B.H. Brlansky, R.F. Lee y Timmer, L.W., eds., University of Florida, Gainesville.

Garzón, T.J.A. (1984). Enfermedad del "permanente" del jitomate (Lycopersicon esculentum Mill.) en Celaya, Gto. 
XI Congreso Nacional de Fitopatología. San Luis Potosí, SLP. Resúmenes Sociedad Mexicana de Fitopatología, A.C. p 138.

Garzón, T.J.A., Cárdenas, V.O.G., Bujanos, M.R., Marín, J.A., Becerra, F.A., Velarde, F.S., Reyes, M.C., González, C.M. \& Martínez, C.J.L. (2009). Asociación de Hemiptera:Triozidae con la enfermedad "permanente del tomate" en México. Agricultura Técnica en México, 35, 58-69. Disponible en línea: http://www.scielo.org.mx/ scielo.php?script $=$ sci_arttext\&pid $=$ S0568251720090001 00006\&lng=es\&nrm=iso

Garzón, T.J.A., Garzón, C.J.A., Velarde, F.S., Marín, J.A. \& Cárdenas, V.O.G. (2005). Ensayos de transmisión del fitoplasma asociado al "permanente del tomate" por el psílido Bactericera cockerelli SULC en México. Entomología Mexicana, 4, 672-674.

Gharalari, A.H., Nansen, C., Lawson, D.S., Gilley, J., Munyaneza, J.E. \& Vaughn, K. (2009). Knockdown mortality, repellency, and residual effects of insecticides for control of adult Bactericera cockerelli (Hemiptera: Psyllidae). Journal of Economic Entomology, 102, 1032 1038. Disponible en línea: https:/www.ncbi.nlm.nih.gov/ pubmed/19610416

Greenway, G.A. \& Rondon, S. (2018). Economic Impacts of Zebra Chip in Idaho, Oregon, and Washington. American Journal of Potato Research, 95, 362. DOI: org/10.1007/ s12230-018-9636-2

Groeneveld, M., Oude, D., Weme, R.G.J., Duurkens, R.H. \& Slotboom, D.J. (2010). Biochemical characterization of the C4-dicarboxylate transporter DctA from Bacillus subtilis. Journal of Bacteriology, 192, 2900-2907. DOI:10.1128/JB.00136-10

Gudmestad, N.C. \& Secor, G.A. (2007). Zebra Chip: A new disease of potato. Nebraska Potato Eyes, 19, 1-4. Disponible en línea: https:/www.ndsu.edu/fileadmin/ potatopathology/ potato_trials/Zebra_Chip_New_ Potato_Disease.pdf

Haapalainen, M., Wang, J., Latvala, S., Lehtonen, M.T., Pirhonen, M. \& Nissinen, A.I. (2018). Genetic variation of 'Candidatus Lineribacter solanacearum' haplotype $\mathrm{C}$ and identification of a novel haplotype from Trioza urticae and stinging Nettle. Phytopathology, 108 (8), 925-934. DOI: 10.1094/PHYTO-12-17-0410-R

Hansen, A.K., Trumble, J.T., Stouthamer, R. \& Paine, T.D. (2008). A new Huanglongbing species, "Candidatus Liberibacter psyllaurous", found to infect tomato and potato, is vectored by the psyllid Bactericerca cockerelli (Sulc). Applied and Environmental Microbiology, 74, 5862- 5865. DOI:10.1128/AEM.01268-08

Henne, D.C., Workneh, F., Wen, A., Price, J.A., Pasche, J.S., Gudmestad, N.C. \& Rush, C.M. (2010). Characterization and epidemiological significance of potato plants grown from seed tubers affected by Zebra Chip disease. Plant Disease, 94, 659-665. DOI: 10.1094/PDIS-94-6-0659
Hernández-Deheza, M.G., Rojas-Martínez, R.I., Rivera-Peña, A., Zavaleta-Mejía, E., Ochoa-Martínez, D.L. \& CarrilloSalazar, A. (2018). Resistance in potato to two haplotypes of 'Candidatus Liberibacter solanacearum'. Journal of Plant Pathology, 100, 191-196. DOI: org/10.1007/ s42161-018-0046-6

Jagoueix, S., Bové, J.M. \& Garnier, M. (1994). The Phloem limited bacterium of greening disease of citrus is a member of the $\alpha$ subdivision of the Proteobacteria. International Journal of Systematic Bacteriology, 44, 379-386. DOI: 10.1099/00207713-44-3-379

Karley, A.J., Douglas, A.E. \& Parker, W.E. (2002). Amino acid composition and nutritional quality of potato leaf phloem sap for aphids. Journal of Experimental Biology, 205, 3009-3018. https://www.ncbi.nlm.nih.gov/ pubmed/12200404

Khairulmazmi, A., Kamaruzaman, S., Habibuddin, H., Jugah, K. \& Syed, O. (2008). Occurrence and spread of Candidatus Liberibacter asiaticus, the causal agent of Huanglongbing disease of citrus in Malaysia. Research Journal of Agriculture and Biological Sciences, 4, 103- 111. https://swfrec.ifas.ufl.edu/hlb/database/pdf/ 00001862.pdf

Lacey, L.A., de la Rosa, F. \& Horton, D.R. (2009). Insecticidal activity of entomopathogenic fungi (Hypocreales) for potato psyllid, Bactericera cockerelli (Hemiptera: Triozidae): Development of bioassay techniques, effect of fungal species and stage of the psyllid. Biocontrol Science and Technology, 19, 957-970. DOI: org/10.1080/09583150903243904

Lacey, L.A., Liu, T.X., Buchman, J.L., Munyaneza, J.E., Goolsby, J.A. \& Horton, D.R. (2010). Entomopathogenic fungi (Hypocreales) for control of potato psyllid, Bactericera cockerelli (Sulc) (Hemiptera: Triozidae) in an area endemic for zebra chip disease of potato. Biological Control, 56, 271-278. DOI: org/10.1016/j. biocontrol.2010.11.012

Lévy, J., Scheuring, D.C., Koym, J.W., Henne, D.C., Tamborindeguy, C., Pierson, E. \& Miller, J.J. (2015). Investigations on Putative Zebra Chip Tolerant Potato Selections. American Journal Potato Research, 92, $417-$ 425. DOI: $10.1007 / \mathrm{s} 12230-015-9452-\mathrm{X}$

Lévy, J. \& Tamborindeguy, C. (2014). Solanum habrochaites, a potential source of resistance against Bactericera cockerelli (Hemiptera: Triozidae) and "Candidatus Liberibacter solanacearum". Journal of Economic Entomology, 107(3), 187-1194 DOI:10.1603/EC13295

Lévy, J., Ravindran, A., Gross, D., Tambrindeguy, C. \& Pierson, E. (2011). Translocation of 'Candidatus Liberibacter solanacearum', the Zebra Chip pathogen, in potato and tomato. Phytopathology, 101, 1285-1291. DOI: org/10.1094/PHYTO-04-11-0121

Li, W., Abad, J.A., French, M.R.D., Rascoe, J.A., Wen, A., Gudmestad, N.C., Secor, G.A., Lee, I.M., Duan, 
Y. \& Lévy, L. (2009). Multiplex real-time PCR for detection, identification and quantification of Candidatus Liberibacter solanacearum in potato plants with zebra chip. Phytopathology, 78, 59-65. DOI: org/10.1016/j. mimet.2009.04.009

Liefting, L.W., Sutherland, P.W., Ward, L.I., Paice, K.L., Weir, B.S. \& Clover, G.R.G. (2009a). A new 'Candidatus Liberibacter' species associated with diseases of solanaceous crops. Plant Disease, 93, 208-214. DOI: org/10.1094/PDIS-93-3-0208

Liefting, L.W., Weir, B.S., Pennycook, S.R. \& Clover, G.R.G. (2009b). 'Candidatus Liberibacter solanacearum', a liberibacter associated with plants in the family Solanaceae. International Journal of Systematic and Evolutionary Microbiology, 59, 2274-2276. DOI: org./10.1099/ijs.0.007377-0

Liefting, L.W., Pérez, E.Z.C., Clover, G.R.G. \& Anderson, J.A.D. (2008). A new 'Candidatus Liberibacter' species in Solanum tuberosum in New Zealand. Plant Disease, 92, 1474. DOI: org/10.1094/PDIS-92-10-1474A

Lin, H., Lou, B., Glynn, J.M., Doddapaneni, H., Civerolo, E.L., Chen, C., Duan, Y., Zhou, L. \& Vahling, C.M. (2011). The complete genome sequence of 'Candidatus Liberibacter solanacearum', the bacterium associated with potato zebra chip disease. PLoS ONE, 6, 1-13. DOI: org/10.1371/journal.pone.0019135

Lin, H., Islam, M.S., Bai, Y., Wen, A., Lan, S., Gudmestad, N.C. \& Civerolo, E.L. (2012). Genetic diversity of 'Candidatus Liberibacter solanacearum' strains in the United States and Mexico revealed by simple sequence repeat markers. European Journal of Plant Pathology, 132, 297-308. https.//doi.org/10.1007/s10658-0119874-3

Linhartová, I., Bumba, L., Mašín, J., Basler, M., Osicka, R., Kamanova, J., Prochazková, K., Adkins, I., HejnovaHolubová, J., Sadílková, L., Morová, J. \& Šebo, P. (2010). RTX proteins: a highly diverse family secreted by a common mechanism. FEMS Microbiology Reviews, 34, 1076-1112. DOI: org/10.1111/j.1574-6976.2010.00231.x

Liu, D. \& Trumble, J.T. (2004). Tomato psyllid behavioral responses to tomato plant lines and interactions of plant lines with insecticides. Journal of Economic Entomology, 97, 1078-1085. DOI: org/10.1093/jee/97.3.1078

Machida, H.R. (2015). Diversity of potato genetic resources. Breeding Science, 65, 26-40. DOI: org/10.1270/ jsbbs. 65.26

Melgoza, V.C.M., León, S.C.R., López, V. J.A., Hernández, E.L.A., Velarde, F.S. \& Garzón, T.J.A. (2018). Presencia de Candidatus Liberibacter solanacearum en Bactericera cockerelli Sulc. asociada con enfermedades en tomate, chile y papa. Revista Mexicana de Ciencias Agrícolas, 9, 499-509. DOI: org/10.29312/remexca.v9i3.267

Mendoza-Herrera, A., Lévy, J., Harrison, K., Yao, J., Ibanez, F. \& Tamborindeguy, C. (2018). Infection by 'Candidatus
Liberibacter solanacearum' haplotypes A and B in Solanumlycopersicum 'Moneymaker'. Plant Disease, 102, 2009-2015. DOI: 10.1094/PDIS-12-17-1982-RE

Morris, J., Shiller, J., Mann, R., Smith, G., Yen, A. \& Rodoni, B. (2017). Novel 'Candidatus Liberibacter' species identified in the Australian eggplant psylid, Acizzia solanicola. Microbial Biotechnology, 10, 833-844. DOI: org/10.1111/1751-7915.12707

Munyaneza, J.E., Sengoda, V.G., Aguilar, E., Bextine, B.R. \& McCue, K.F. (2013). First Report of 'Candidatus Liberibacter solanacearum' Infecting Eggplant in Honduras. Plant disease, 97(12), 1654. DOI:10.1094/ PDIS-06-13-0641-PDN

Munyaneza, J.E. (2012). Zebra chip disease of potato: Biology, epidemiology, and management. American Journal of Potato Research, 89, 329-350. DOI: org/10.1007/ s12230-012-9262-3

Munyaneza, J.E., Buchman, J.L., Sengoda, V.G., Fisher, T.W. \& Pearson, C.C. (2011). Susceptibility of selected potato varieties to zebra chip potato disease. American Journal of Potato Research, 88, 435-440. DOI: org/10.1007/ s12230-011-9209-0

Munyaneza, J.E., Fisher, W.J., Sengoda, G.V. \& Garczynski, F. (2010). First report of "Candidatus Liberibacter solanacearum" associated with psyllid affected carrots in Europe. Plant Disease, 94, 639. DOI: org/10.1094/PDIS94-5-0639A

Munyaneza, J.E., Sengoda, V.G., Crosslin, J.M., Garzón, T.J.A. \& Cárdenas, V.O.G. (2009a). First Report of "Candidatus Liberibacter solanacearum" in tomato plants in Mexico. Plant Disease, 93, 1076. DOI: org/10.1094/ PDIS-93-10-1076A

Munyaneza, J.E., Sengoda, V.G., Garzón, T.J.A. \& Cárdenas, V.O.G. (2009b). First report of "Candidatus Liberibacter solanacearum" in pepper plants in Mexico. Plant Disease, 93, 1076. DOI: org/10.1094/PDIS-93-10-1076B

Munyaneza, J.E., Sengoda, V.G., Crosslin, J.M., De la Rosa, L.G. \& Sánchez, A. (2009c). First Report of 'Candidatus Liberibacter psyllaurous' in Potato Tubers with Zebra Chip Disease in Mexico. Plant Disease, 93, 552. DOI: org/10.1094/PDIS-93-5-0552A

Munyaneza, J.E., Crosslin, J.M. \& Upton, J.E. (2007a). Association of Bactericera cockerelli (Homoptera: Psyllidae) with "zebra chip", a new potato disease in southwestern United States and Mexico. Journal of Economic Entomology, 100, 656-663. DOI: org/10.1603/0022-0493(2007)100[656:AOBCHP]2.0. $\mathrm{CO} ; 2$

Munyaneza, J.E., Goolsby, J.A., Crosslin, J.M. \& Upton, J.E. (2007b). Further evidence that zebra chip potato disease in the lower Rio Grande Valley of Texas is associated with Bactericera cockerelli. Subtropical Plant Science, 59, 30 37. Disponible en línea: www.subplantsci.org/wp-content/ uploads/2016/02/SPSJ-59-30-37-Munyaneza-et-al.pdf 
Nachappa, P., Lévy, J. \& Tamborindeguy, C. (2012). Transcriptome analyses of Bactericera cockerelli adults in response to "Candidatus Liberibacter solanacearum" infection. Molecular Genetics and Genomics, 287, 803817. DOI:org/10.1007/s00438-012-0713-9

Nelson, W.R, Tonja, W.T., Fisher, \& Munyaneza, J.E. (2011). Haplotypes of "Candidatus Liberibacter solanacearum" suggest long-standing separation. European Journal of Plant Pathology, 130, 5-12. DOI: org/10.1007/s10658010-9737-3

Nyilasi, I., Papp, T., Csernetics, A., Krizsán, K., Nagy, E. \& Vágvölgyi, C. (2008). High-affinity iron permease (FTR1) gene sequence-based molecular identification of clinically important Zygomycetes. Clinical Microbiology and Infection, 14, 393-397. DOI: org/10.1111/j.14690691.2007.01932.x

Pavlista, A.D. (2002). Potato (tomato) psyllids. Nebraska Potato Eyes, 14,1-4. Disponible en línea: https:// cropwatch.unl.edu/potato/potato psyllids

Peng, L., Trumble, J.T., Munyaneza, J.E. \& Liu, T.X. (2011). Repellency of a kaolin particle film to potato psyllid, Bactericera cockerelli (Hemiptera: Psyllidae), on tomato under laboratory and field conditions. Pest Management Science, 67, 815-824. DOI: org/10.1002/ps.2118

Pletsch, D.J. (1947). The potato psillid Paratrioza cockerelli (Sulc), its biology and control. Montana Agricultural Experiment Station. Boletín, 446. Bozeman, MT, U.S. 1-95pp.

Ravindran, A., Lévy, J., Pierson, E. \& Gross, D.C. (2011). Development of primers for improved PCR detection of the potato zebra chip pathogen, 'Candidatus Liberibacter solanacearum'. Plant Disease, 95, 1542-1546. DOI: org/10.1094/PDIS-05-11-0386

Ravindran, A., Lévy, J., Pierson, E. \& Gross, D.C. (2015). Loop-Mediated Isothermal Amplification Procedure (LAMP) for Detection of the Potato Zebra Chip Pathogen "Candidatus Liberibacter solanacearum". Christophe Lacomme (ed.), Plant Pathology: Techniques and Protocols, Methods in Molecular Biology, 1302, 85-97. DOI:10.1007/978-1-4939-2620-6_7.

Ravindran, A., Saenkham, P., Lévy, J., Tamborindeguy, C., Lin, H., Grossm, D.C. \& Pierson, E. (2017). Characterization of the Serralysin-like gene of ' $\mathrm{Ca}$. Liberibacter solanacearum' associated with potato zebra chip disease. Phytopathology, 108, 327-335. DOI: org/10.1094/PHYTO-02-17-0064-R

Rojas-Martínez, R.I., Camacho-Tapia, M., Zavaleta, M.E. \& Lévy, J. (2016). First report of the presence of haplotypes A and B of Candidatus Liberibacter solanacearum in chili (Capsicum annuum L.) in the Central Region of Mexico. Journal of Plant Pathology, 98, 111-115. Disponible en línea: www.jstor.org/stable/24892629

Rubio, C.O., Almeyda, L.I., Ireta, M.J., Sánchez, S.J., Fernández, S.R., Borbón, S.J., Díaz, H.C., Garzón, T.J.,
Rocha, R.R. \& Cadena, H.M. (2006). Distribution of potato purple top and Bactericera cockerelli Sulc. in the main potato production zones in Mexico. Agricultura Técnica en México, 32, 201-211. Disponible en línea: http://www. scielo.org.mx/pdf/ agritm/ v32n2/v32n2a8.pdf

Rubio-Covarrubias, O.A., Cadena-Hinojosa, M.A., Prager, S.M., Wallis, C.M. \& Trumble, J.T. (2017). Characterization of the tolerance against zebra chip disease in tubers of advanced potato lines from Mexico. American Journal of Potato Research, 94,342-356 DOI: 10.1007/s12230-017-9570-8

Santos-Cervantes, M.E., Chávez-Medina, J.A., MéndezLozano, J. \& Leyva-López, N.E. (2008). Detection and molecular characterization of two little leaf phytoplasma strains associated with pepper and tomato diseases in Guanajuato and Sinaloa, Mexico. Plant Disease, 92, 1007-1011. DOI: 10.1094/PDIS-92-7-1007

Sechler, A., Schuenzel, E.L., Cooke, P., Donnua, S., Thaveechai, N., Postnikova, E., Stone, A.L., Schneider, W.L., Damsteeqt, V.D. \& Schaad, N.W. (2009). Cultivation of 'Candidatus Liberibacter asiaticus', 'Ca. L. africanus' and 'Ca. L. americanus' associated with huanglongbing. Phytopathology, 99, 480-486. DOI:10.1094/PHYTO-99-5-0480

Secor, G.A. \& Rivera, V.V. (2004). Emerging diseases of cultivated potato and their impact. Revista Latinoamericana de la Papa, 1, 1-8. Disponible en línea: https://www.researchgate.net/publication/284255582 Emerging_diseases_of_cultivated_potato_and_their_ impact_on_Latin_America

Secor, G.A., Rivera, V.V., Abad, I.M., Lee, G.R.G., Clover, L.W., Liefting, X., Li, \& De Boer S.H. (2009). Association of 'Candidatus Liberibacter solanacearum' with zebra chip disease of potato established by graft and psyllid transmission, electron microscopy, and PCR. Plant Disease, 93, 574-583. DOI: org/10.1094/PDIS-936-0574

Shrivastava, R. \& Miller, J.F. (2009). Virulence factor secretion and translocation by Bordetella species. Current Opinion in Microbiology, 12, 88-93. DOI:10.1016/j. mib.2009.01.001

Swisher, K.D., Munyaneza, J.E., Velásquez-Valle, R. \& Mena-Covarrubias, J. (2018). Detection of pathogens associated with psyllids and leafhoppers in Capsicum annuum L. in the Mexican states of Durango, Zacatecas, and Michoacan, Plant Disease 102(1), 146-153. DOI: 10.1094/PDIS-05-17-0758-RE

Tanaka, F.A.O., Colleta, F.H.D., Alves, K.C.S., Spinelli, M.O., Machado, M.A. \& Kitajima, E.W. (2007). Detection of the "Candidatus Liberibacter americanus" in phloem vessels of experimentally infected Catharanthus roseus by scanning electron microscopy. Fitopatologia Brasileira, 36, 519-520. DOI: 10.1590/ S0100-41582007000600013 
Teresani, G.R., Bertolini, E., Alfaro, F.A., Martínez, C., Tanaka, F.A.O. \& Kitajima, E.W., (2014). Association of 'Candidatus Liberibacter solanacearum' with a vegetative disorder of celery in Spain and development of a Real-Time PCR method for its detection. Phytopathology, 104, 804-811. DOI: 10.1094/phyto-0713-0182-r 23

Texeira, C.D., Saillard, C., Eveillard, S., Danet, L.J., Da Costa, P., Ayres, J.A. \& Bové, J. (2005). “Candidatus Liberibacter americanus", associated with citrus huanglongbing (greening disease) in Saõ Paulo State, Brazil. International Journal of Systematic and Evolutionary Microbiology, 55, 1857-1862. DOI:10.1099/ijs.0.63677-0

Torres, G.L., Cooper, W.R., Horton, D.R., Swisher, K.D., Garczynski, S.F., Munyaneza, J.E. \& Bárcenas, N.M. (2015). Horizontal Transmission of "Candidatus Liberibacter solanacearum" by Bactericera cockerelli (Hemiptera: Triozidae) on Convolvulus and Ipomoea (Solanales: Convolvulaceae). PLoS ONE, 10, e0142734. DOI:10.1371/journal.pone.0142734

Villechanoux, S., Garnier, M. \& Bové, J.M. (1990). Purification of the bacterium-like organism associated with greening disease of citrus by immune affinity chromatography and monoclonal antibodies. Current Microbiology, 21, 175180. DOI: org/10.1007/BF02092118

Viola, R., Roberts, A.G., Haupt, S., Gazzani, S., Hancock, R.D, Marmiroli, N., Machray, G.C. \& Oparka, K.J. (2001). Tuberization in potato involves a switch from apoplastic to symplastic phloem unloading. Plant Cell, 13, 385-398. DOI: 10.1105/tpc.13.2.385

Wang N., Pierson E.A., Setubal J.C., Xu J., Julien G.L., Zhang Y., Li, J., Thiberio, R. \& Martins, L.J. (2017). The Candidatus Liberibacter-Host Interface: Insights into Pathogenesis Mechanisms and Disease Control. Annual Review of Phytopathology, 55, 451-482. DOI: org/10.1146/annurev-phyto-080516-035513

Yang, X.B., Zhang, Y.M., Hua, L., Peng, L.N., Munyaneza, J.E. \& Liu, T.X. (2010). Repellency of selected biorational insecticides to potato psyllid, Bactericera cockerelli (Hemiptera: Psyllidae). Crop Protection, 29, 1329-1324. DOI: org/10.1016/j.cropro.2010.06.013

Yao, J., Saenkham, P., Lévy, J., Ibanez, F., Noroy, C., Mendoza, A., Huot, O., Meyer, D.F., \& Tamborindeguy,C. (2016). Interactions "Candidatus Liberibacter solanacearum" Bactericera cockerelli:Haplotype effect on vector fitness and gene expression analyses. Frontiers in Cellular and Infection Microbiology, 6(62), 1-13. DOI: 10.3389/ fcimb.2016.00062

Yurgel, S.N. \& Kahn, M.L. (2004). Dicarboxylate transport by rhizobia. FEMS Microbiology Reviews, 28, 489-501. DOI: org/10.1016/j.femsre.2004.04.002

Zhang, M.Q., Powell, C.A., Zhou, L.J., He, Z.L., Stover, E. \& Duan, Y.P. (2011). Chemical compounds effective against the citrus Huanglongbing bacterium 'Candidatus Liberibacter asiaticus' in planta. Phytopathology, 101(9), 1097-1103. DOI:10.1094/PHYTO-09-10-0262 